\title{
Optical Image Compression Using a Real Fourier Plane
}

\author{
Abdulsalam G. Alkholidi \\ Faculty of Engineering, Electrical Engineering Department, Sanaa University, Sanaa, Yemen \\ Email: Abdulsalam.alkholidi@gmail.com
}

Received April 7, 2013; revised May 7, 2013; accepted May 17, 2013

Copyright (C) 2013 Abdulsalam G. Alkholidi. This is an open access article distributed under the Creative Commons Attribution License, which permits unrestricted use, distribution, and reproduction in any medium, provided the original work is properly cited.

\begin{abstract}
Hastening transmission by efficiently providing compression is our goal in this work. Image compression consists in reducing information size representing an image. Elimination of redundancies and non-pertinent information enables memory space minimization and thus fast data transmission. Optics can offer an alternative choice to overcome the limitation of numerical compression algorithms. In this paper, we propose real-time optical image compression using a real Fourier plane to save time required for compression by using the principles of coherent optics. Digital and optical simulation results are presented and analyzed. An optical compression decompression setup is demonstrated using two different SLMs (SEIKO and DisplayTech). The purpose of this method is to simplify our earlier method, improve the quality of reconstructed image, and avoid the disadvantages of numerical algorithms.
\end{abstract}

Keywords: FT; Optical Compression; a Real Fourier Plane; Hologram; SLM

\section{Introduction}

In recent years, there has been much interest in image compression algorithms which are proposed in this paper. Our algorithm is oriented towards large size images. The goal of this work is to minimize the time required for compression with high compression ratio $\left(C_{r}\right)$. One of the current technological challenges in image compression is to achieve real-time imaging rates with a minimal sacrifice in signal-to-noise ratio (SNR) [1]. We propose a novel technique of image compression using a real Fourierplane capable of real-time image compression. In the frame of the present work our objective is to simplify our earlier optical image compression algorithm using the JPEG and optical JPEG standards [2]. We intend to eliminate the most complex part in the synoptic diagrams of the set up implementation compression and decompression described in $[2,3]$.

To digitally decompress large images, they should be first transmitted to a calculator to enable applying one or several digital compression methods. The real-life application of this technique; consists first in optically compressing these images. Starting from an image, with size $(N \times N)$ pixels (Figure 1(a)), we obtain a compressed version of only $(c \times c)$ pixels as pointed out by Figure 1(f). This results in a decrease of the volume of information to be transmitted to the calculator. The latter may provide additional compression, by applying digital methods. In this paper, we provide a detailed technical de- scription of the proposed architecture. This exposition is interested to serve as a reader-friendly starting point for those interested in learning about optical JPEG compression. Although many details are included in our presentation, some details were necessarily omitted to focus on the main subject that is compression. The reader should, therefore, refer to standards of optical JPEG compression [2,3] before attempting an implementation. Indeed, much work has been done toward developing compression methods, most techniques reported, such as presented in [4-8].

The remainder of this paper is structured as follows: Section 2 provides a brief overview of the optical JPEG standards. This is followed, in Section 3, by a detailed description of mathematical approach of the proposed algorithm. In Section 4, optical architecture of compression/decompression is presented. Section 5, an optical setup of image compression/decompression of proposed method is presented and the results are presented in Section 6. Finally, we conclude with some closing remarks in Section 7.

\section{Optical JPEG Compression}

By using the principle of coherent optics, optical JPEG (OJPEG) compression and decompression; process the whole image at once and do not divide it into blocks. The disadvantage of OJPEG is the fact that its optical implementation is complicated $[2,3]$. To relax this constraint, 


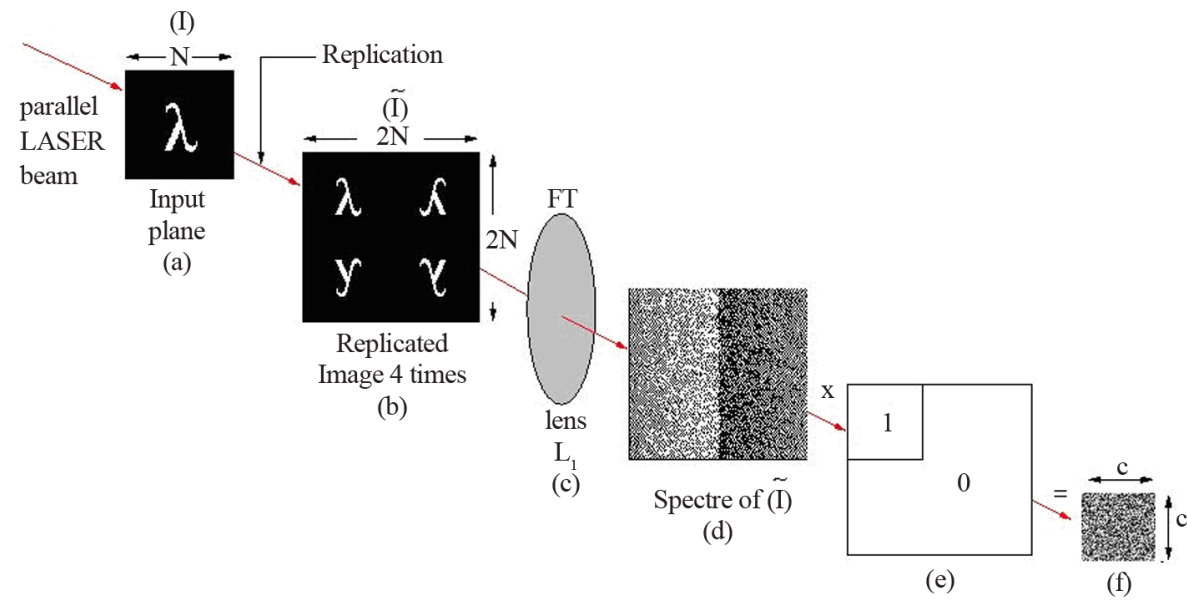

$(\mathrm{c} \times \mathrm{c})$ pixels is a compression results using a real Fourier plane in the frequency domain. $=($ the number of transmitted pixels)

Figure 1. Synoptic diagram of the setup implementation of the optical image compression using a real Fourier plane, (a) original image $I(N \times N)$ pixels, (b) replicated image $I(2 N \times 2 N)$ pixels, (c) convergent lens to calculate FT, (d) real spectrum of replicated image, $(\mathrm{e})$ information selection, $(\mathrm{f})(c \times c)$ pixels is the size of compressed spectrum to be transmitted.

we propose a new optical image compression method using a real Fourier plane.

\section{Mathematical Approach of Optical Image Compression Using a Real Fourier Plane}

The main steps to compress an image in this work are as follows:

1) Replicate the original image $(N \times N)$ pixels as showing in Figure 2. This operation comes down to making the image even. We then obtain a $(2 N \times 2 N)$ pixels image with hermitian symmetry. Since the image is initially real, its hermetian version is merely even.

2) By applying Equation (1) to an image of $(2 N \times 2 N)$ pixels, we obtain Equation (2).

Equation (1) gives the discrete version of the FT referred to as the 2 dimensional DFT of an $(N \times N)$ pixels image.

$$
\begin{aligned}
& F(p, q)= \\
& \frac{1}{N \times N} \sum_{m=0}^{N-1} \sum_{n=0}^{N-1} f(m, n) \times \mathrm{e}^{-j 2 \pi\left(\left(\frac{m p}{N}\right)+\left(\frac{n q}{N}\right)\right)}
\end{aligned}
$$

with

$$
\begin{aligned}
& p \in 0 \text { to }[N-1] \\
& q \in 0 \text { to }[N-1]
\end{aligned}
$$

The term $f(m, n)$ stands for the discrete version of the two-dimensional signal $f(x, y)$.

For $N=2^{k}, k$ is an integer, fast algorithms FFT to compute DFT exist. For the obtained hermetian image, the DFT is expressed as follows:

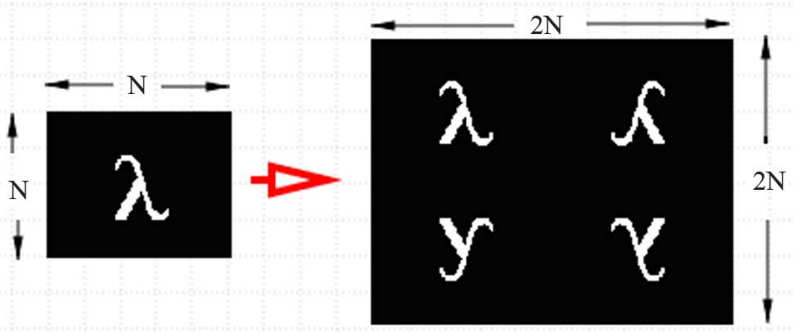

Figure 2. Characteristic replication of the input image.

$$
\tilde{F}(p, q)=\frac{1}{4 N \times N} \sum_{m=0}^{2 N-1} \sum_{n=0}^{2 N-1} \tilde{f}(m, n) \times \mathrm{e}^{-j 2 \pi\left(\left(\frac{m p}{2 N}\right)+\left(\frac{n q}{2 N}\right)\right)}
$$

with

$$
\begin{aligned}
& p \in 0 \text { to }[2 N-1] \\
& q \in 0 \text { to }[2 N-1]
\end{aligned}
$$

3) The real spectrum obtained using Equation (2) after replication of the image Figure 3(d), is then quantified.

We note that the input image with $(N \times N)$ pixels presented in Figure 3(a) is real whereas its Fourier transform is complex. The main difference between the OJPEG compression presented in [2,3] and the proposed compression technique is that the spectrum obtained of replicated image $(2 N \times 2 N)$ pixels showing in Figure 2 is complex for the first one as presented in Figures 3(a) and (c), because we presented a mathematical approach for this objective demonstrated in [3]. If we replicate an image to obtain $(2 N \times 2 N)$ pixels, then its Fourier transform is real even. This presents the core of this work; that is,

$$
F(u, v)=F^{*}(-u,-v)
$$




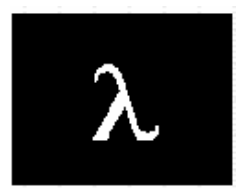

(a)

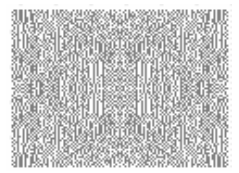

(c)

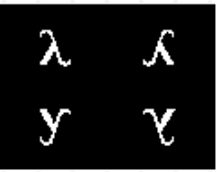

(b)

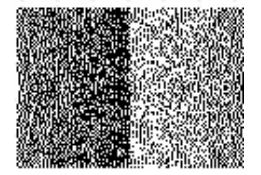

(d)
Figure 3. (a) Input image, symbol lambda $\mathrm{I}(N \times N)$ pixels, (b) replicated image with $I((2 N \times 2 N)$ pixels, (c) complex hermetian spectrum obtained from (a), (c) real even spectrum obtained from (b).

where "*" indicates the standard conjugate operation on complex number. From this, it follows that:

$$
|F(u, v)|=|F(-u,-v)|,
$$

Which says that the spectrum of the Fourier is symmetric $[10,11]$. The two spectra (c) and (d) are symmetric. But spectra (c) is symmetric complex and spectra (d) is symmetric real even.

4) After the real Fourier spectra step, we proceed to the quantification and selection. It consists firstly in mapping the spectra values on a set of selected values, (quantization), and secondly in eliminating redundant information in this spectra plane. These operations are performed together, thanks to a single threshold:

The quantification: this operation consists of dividing the values of the spectra by another matrix containing quantization values. The optical implementation of this operation is performed in the spectral domain by multiplying the spectrum coefficient by another computer generated hologram containing values of quantization. The filtering: to increase the compression ratio after quantization, a threshold operation is used to retain only the part of information that we want to transmit. This operation corresponds in general at a low-pass filter to preserve the low frequency information which is relevant for the spectrum obtained. High frequencies are omitted. The filter size is then reduced to (c), expressed as follows:

$$
c=\sqrt{\frac{N \times N}{Q_{s}}}
$$

- $c \times c$ is the size of compressed spectrum to be transmitted,

- $Q_{s}$ is the quantification steps (in change the intensity of laser's energy).

Two compression parameters can be defined:

$$
T_{i}=\frac{100 \times C \times C}{N \times N} \%=\frac{100}{Q_{s}}
$$

$$
C_{r o}=\left(100-T_{i}\right) \%
$$

- $T_{i}$ is the transmitted information, percentage,

- $C_{r o}$ is the optical compression rate.

The compression rate for digital image compression technique is defined as follows:

$$
C_{r}=\frac{\text { Original Image Size }}{\text { Compressed Image Size }}
$$

The main difference between two compression techniques is digital-JPEG reduces the number of bytes while optical image compression, using a real Fourier plane the number of pixels, is reduced as seen in Equations (7) and (8).

The optical compression ratio $C_{r o}$ mentioned above Equation (7) consists in comparing the number of pixels remaining after compression with that of the initial image. As this ratio takes into account the remaining number of pixels, thus it is completely normal that the quality of the image is degraded when the compression ratio increases. The increase in the ratio means that there are fewer of pixels to be transmitted and thus less information on the image.

\section{Optical Image Compression and Decompression Architectures Using a Real Fourier-Plane}

\subsection{Architecture of Optical Compression}

We propose an optical architecture in a real Fourier plane by using a DFT. This optical compression technique can be processed numerically or optically. The synoptic diagram of the optical setup is demonstrated in Figure 1.

The synoptic diagram presented in Figure 1 represents successive operations. The first one consists of replicating the input image Figure 1(a) in a specific way Figure 1(b). The objectives of this replication operation are:

1) To obtain a real spectrum easy to compress in the frequency domain as illustrated in Equations (6) and (7).

2) To avoid the noise order of zero generated by the beam of LASER as demonstrated in Figure 4 decompressed image.

The second stage performs the FT of this duplicated image by using a convergent lens " $\mathrm{L}_{1}$ " as shown in Figure 1(c). The real spectrum Figure 1(d) is multiplied by the low-pass filter of Figure 1(e) to increase the compression ratio after quantization. In fact, this filter is used for the purpose of preserving the low frequency, and to select the transmitted data and to obtain the compressed optical part of information the input image. Figure 1(f), this spectra, of size $(c \times c)$ pixels (see Equation (5)), contains the data to be transmitted.

\subsection{Architecture of Optical Decompression}

On the receiver side, transmitted data should be decom- 
pressed to obtain the original image. The decompression consists in reversing the operations carried out during compression side. For this purpose, we propose the synoptic diagram of Figure 5. Information at the receiver is restricted to a $(c \times c)$ pixels array Figure 5(a), then we should first restore a $(2 N \times 2 N)$ pixels spectrum array by filling zero padding the remaining zone by zeros as indicated in Figure 5(b). We take the conjugate version of the image of Figure 5(a) and insert two duplicates of it in the image as illustrated in Figure 5(b), where " $c$ " is indicated. Two duplicates of the image of Figure 5(a), itself are placed in the two zones where " $c$ " is indicated in Figure 5(b).

Each two duplicates in the diagonals of Figure 5(b) are point wise symmetrical with respect to the central point of the $(2 N \times 2 N)$ pixels image. The objective of this reconstruction is to obtain the spectrum of the image $(2 \mathrm{~N}$ $\times 2 N)$ as shown in Figure 5(c). Finally, an optical Inverse Fourier Transform (IFT) is obtained by a convergent lens " $\mathrm{L}_{2}$ " Figure 5(d)) which yields to the replicated image. Selecting one of the four quadrants gives the decompressed original image as showing in Figure 5(e).

\section{Implementations of Optical Image Compression/Decompression Using a Real Fourier Plane}

\subsection{Compression Implementation}

After illustrating the formulation and compression/decompression architectures of optical image compression using a real Fourier plane, we turn to the optical implementation of the technique. For this purpose, we will present an all-optical setup which contains two stages of our method image compression/decompression. The diagram

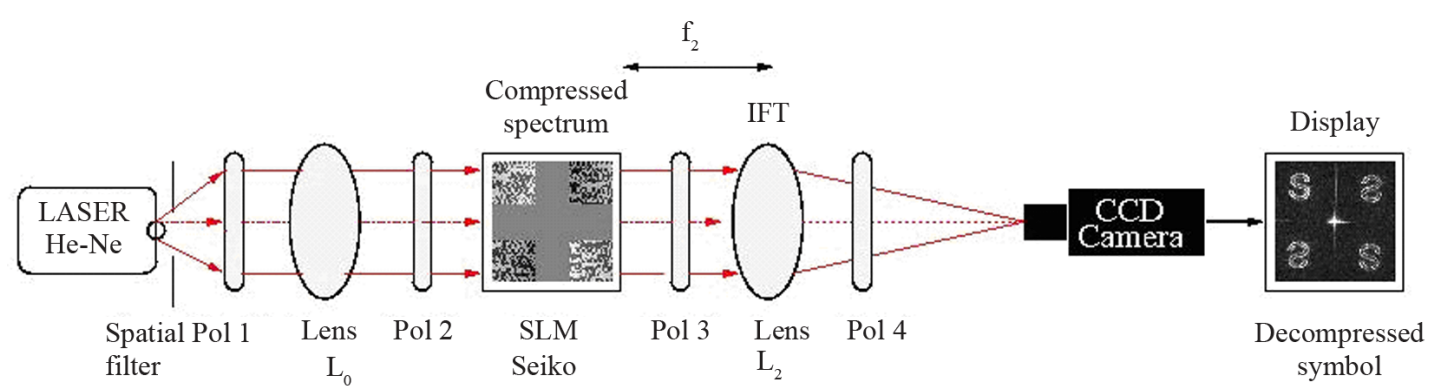

Figure 4. The optical setup decompression using SEIKO SLM.

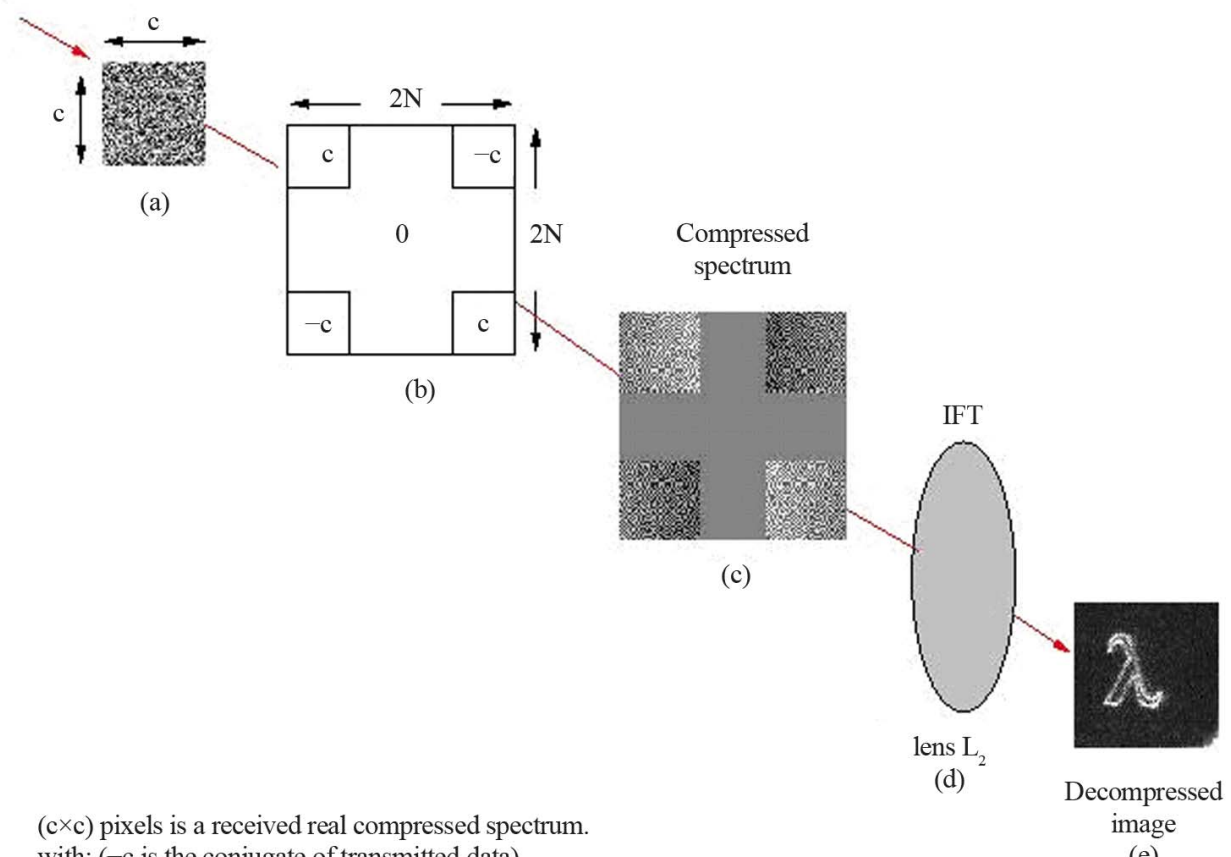

with: ( $-\mathrm{c}$ is the conjugate of transmitted data)

(e)

Figure 5. Synoptic diagram of the optical image decompression using a real Fourier plane, (a) received information, (b) restore a $(2 N \times 2 N)$ pixels spectrum array by filling zero padding the remaining zone by zeros, (c) conjugate version of the image of Figure 5(a) and insert two duplicates of it in the image Figure 5(b) where "c" is indicated, (d) convergent lens to calculate IFT, (e) decompressed image. 
of the optical compression setup of the proposed method is presented in Figure 6.

Technical characteristics of this montage of optical image compression using a real Fourier plane. The montage presented in Figure 6 consists of the following dispositive:

A monochromatic light source LASER He-Ne source $(\lambda=633 \mathrm{~nm}, 25 \mu \mathrm{W})$, Spatial Filter, Three separators cubes, two polarizers, two mirrors, two lens $\mathrm{L}_{0}, \mathrm{~L}_{1}$, the first one its focal is of $160 \mathrm{~mm}$ to be collimated the laser's beam and the second one to realize the Fourier transform. Compression implementation setup consists tow Spatial Light Modulators (SLM). A SLM generally consists of an addressing material and a modulating material. The optical property of the modulating material is changed by write-in information, and phase or amplitude of readout light is modulated in parallel, corresponding to the write-in information [12].

The first one SLM-1 of $640 \times 480$ pixels from Seiko with resolution of $42 \mu \mathrm{m}$ is used to display the input plane of size $(2 N \times 2 N)=(256 \times 256)$ pixels as demonstrated in Figure 6 (input plane). This VGA3 is a high resolution SLM based on Thin Film Transistor (TFT) twisted nematic (TN) display. It provides grey scale displaying capability at standard video frame rates. The second SLM-2 of $1280 \times 780$ pixels, from Display Tech to display the hologram necessary to realize the low pass filter (information selection).

The compression plane (output plane) consists of camera CCD of size $753 \times 582$ pixels with a resolution of $6 \mu \mathrm{m}[13]$.

The first step for proposed optical montage is to calculate the focal distance $\left(\mathrm{L}_{1}\right)$ of the Fourier lens. For that, we are going to utilize the following equation:

$$
f_{1}=N \times d_{e} \times d_{f} / \lambda
$$

where $\lambda$ is the operating beam wavelength, $f_{1}$ is the focal distance of $\mathrm{L}_{1}, N$ is the number of pixels of the various planes, $d_{i}$ and $d_{o}$, respectively, represent the size of the pixel (resolution) of the input and output planes.

$$
\begin{aligned}
f_{1} & =\frac{N \times d_{e} \times d_{f}}{\lambda} \\
& =\frac{256 \times 42 \times 10^{-6} \times 13.2 \times 10^{-6}}{633 \times 10^{-6}}=224.212 \mathrm{~mm}
\end{aligned}
$$

After having described the different components utilized in this optical setup presented in Figure 6, we are going to present the optical compression results. One of the disadvantages of this implementation is difficult to align the optical montage. The results of this multiplication, we obtain the spectra of compressed image $(c \times c)$ pixels. That is registered using a CCD camera. The obtained result considers a numerical version of optical image compression using a real Fourier plane. The photo of the setup is given in Figure 7.

Figure 8 shows the image of size $(c \times c)$ pixels that considers the result of optical compression implementation using a real Fourier plane, contains the data to be transmitted.

\subsection{Decompression Implementation}

After illustrating the optical compression implementation stage, we turn to the optical decompression setup. For this purpose, we will present an all-optical setup consisting of different components for implementing the image decompression using a real Fourier plane using two different SLMs. In this implementation, we are going to implement two spectra:

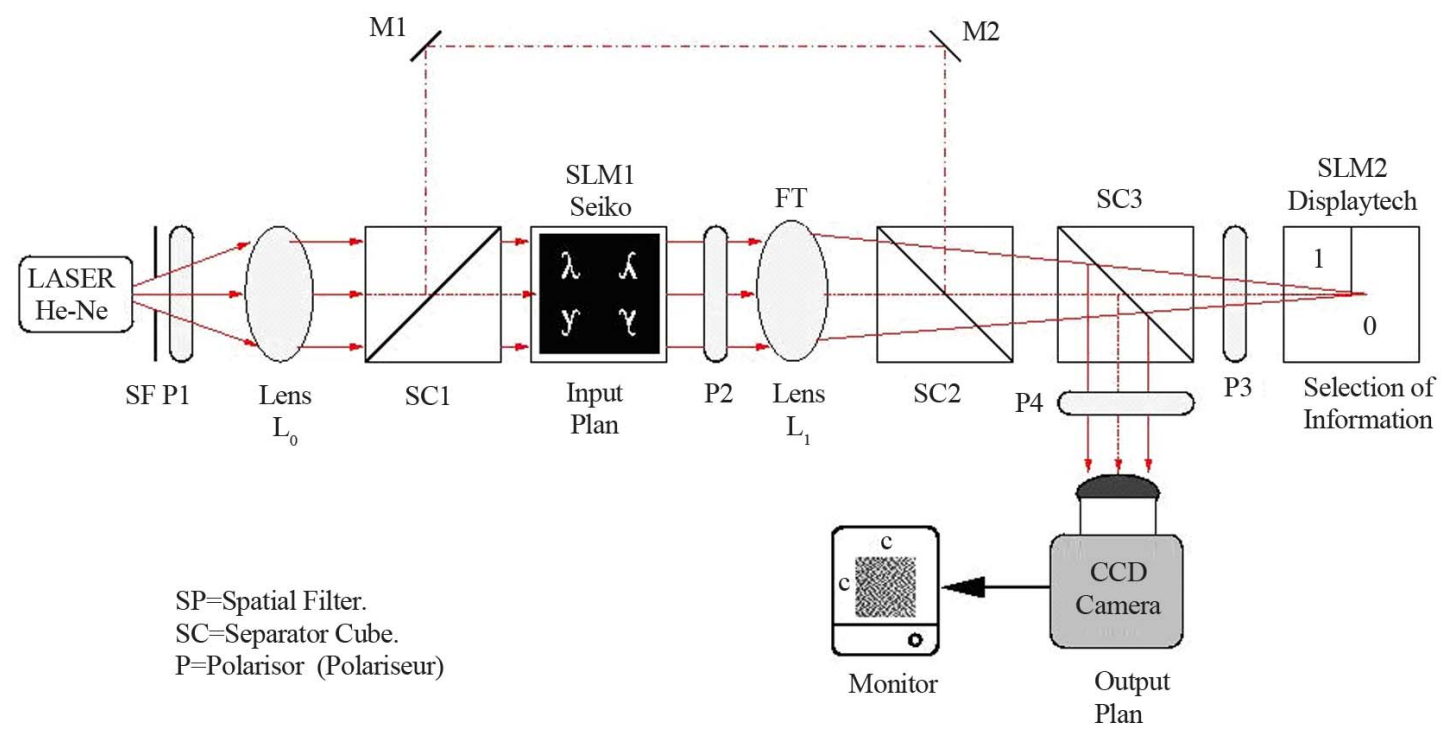

Figure 6. The optical setup used to optical image compression using a real Fourier plane. 


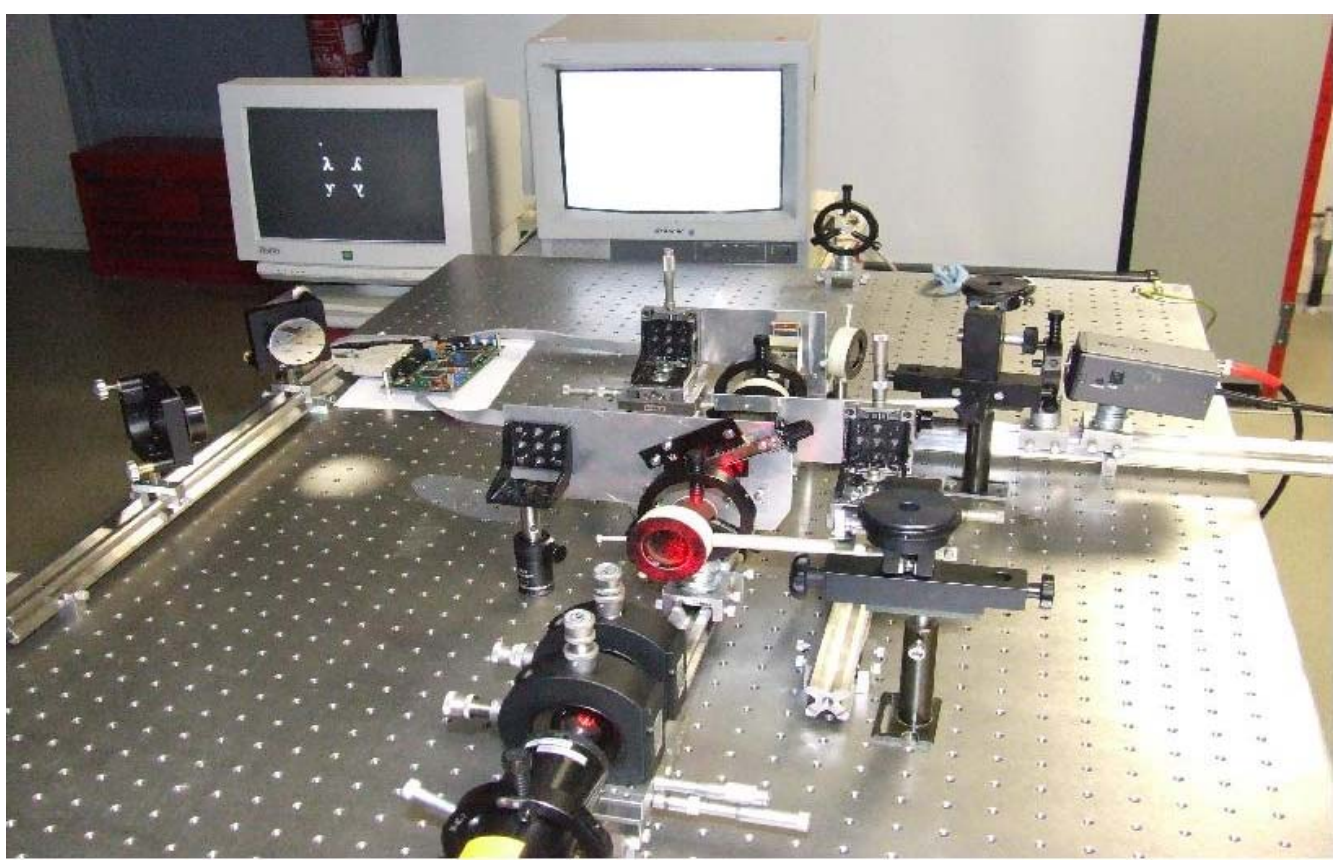

Figure 7. A photo of the setup used for the optical compression stage.

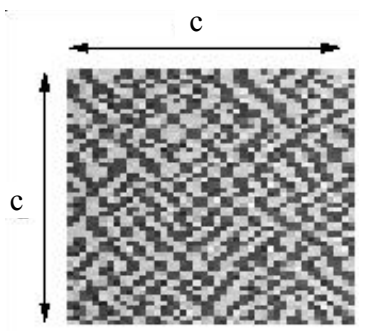

Figure 8. The result of optical compression implementation using a real Fourier plane.

- Compressed spectrum in grey level as demonstrated in Figure 4, where, we used SLM SEIKO.

- Binary compressed spectrum as demonstrated in Figure 9, where, we used DisplayTech SLM.

We used two SLM with different technical characteristics to compare the decompressed images. In fact, firstly we used the modulator SEIKO permits to display an image with several levels of grey, but with a weaklevel of quality of reconstructed image. Secondly, we used DesplayTech binary modulator that displays revenue so important, but it does not permits to the display of the specters with several levels of grey. For this purpose, we propose the synoptic diagram of Figure 4. Information at the receiver is restricted to $(c \times c)$ pixels. Two polarizers are placed before and after the SLM in order to operate in phase. A mask is used to isolate only the additive share of the SLM. The modulator is used to display the hologram that includes $(c \times c)$ pixels representing the compressed spectrum of the image obtained digitally (Figures 10(a) and (b)).

\section{Results of Optical Implementation}

To validate the principle of proposed compression/decompression method of a real Fourier plane, we have considered several images as shown in Figure 11.

In column (a), we see the different symbols and an image used to validate our optical implementation of compression/decompression stages. Four images are considered including three binary letters images, namely " $\lambda$ ", "S", and " $\Psi$ " and, a gray level image, namely the famous "Lena". Each input image extends over $128 \times 128$ pixels. In column (b), we have replicated each input image four times. Indeed, each image is mirrored horizontally, vertically and obliquely. In column (c), the calculated Optical Fourier Transform (OFT) of the different replicas is shown. We have optically implemented the IFT of these various spectra. These are used as references. The results are given in column (d). In column (e), we see the different compressed spectra with our proposed method. Finally, in column (f) the optically reconstructed versions of decompressed images are shown. The results, shown in column " $\mathrm{f}$ ", validate the principle of our implementation method of the optical image compression/decompression using a real Fourier plane.

Figure 12 shows the optical implementation of the compression/decompression of proposed method. The input plane of this setup consists of a binary modulator DisplayTech to display the binary compressed spectra as demonstrated in Figure 12(e), (a) original image; (b) replicated image; (c) replicated image spectra; (d) optical output plane of the replicated image spectra; (e) compressed spectra; (f) decompressed image. Finally, we 


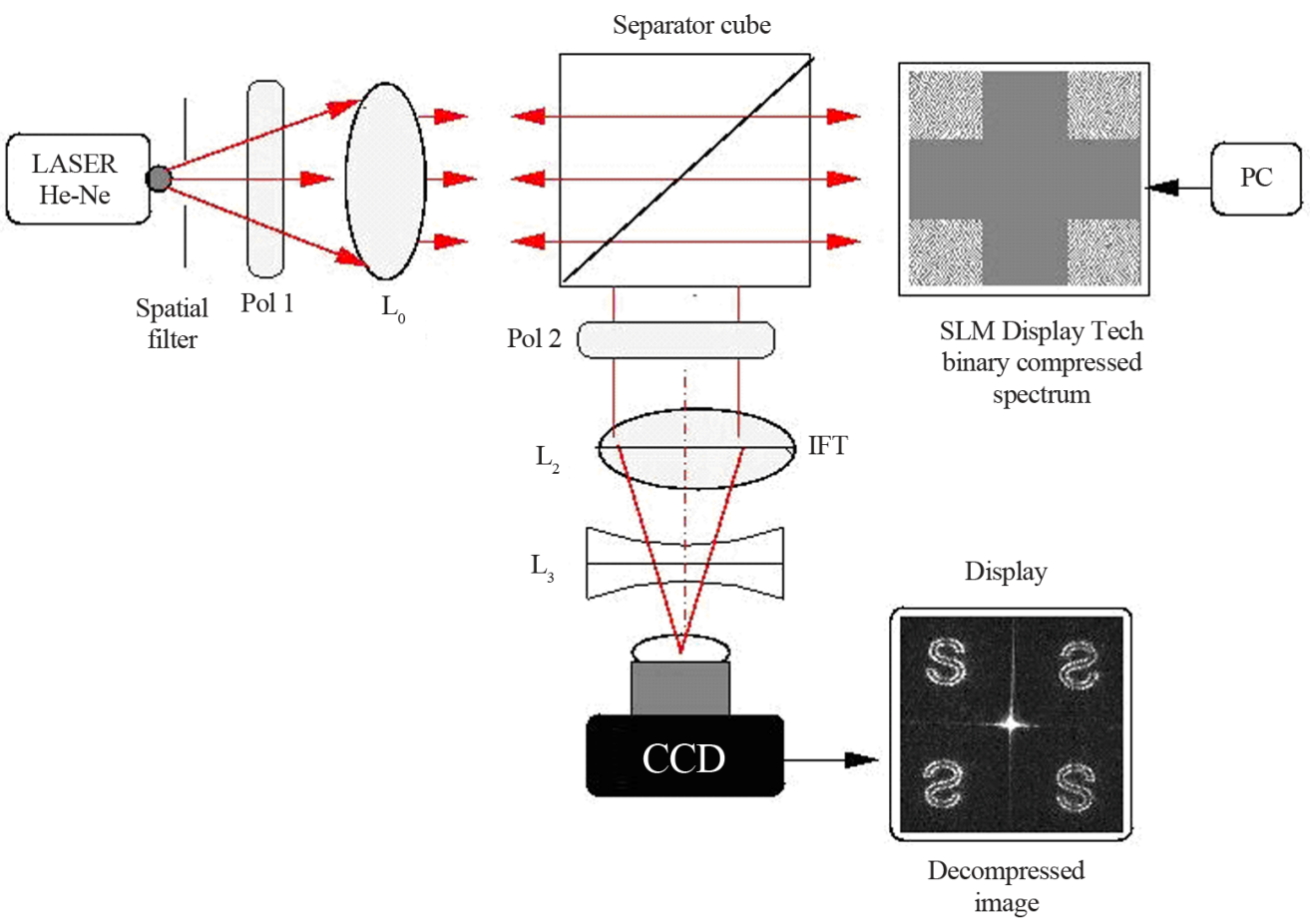

Figure 9. The optical setup decompression using DisplyTech SLM.

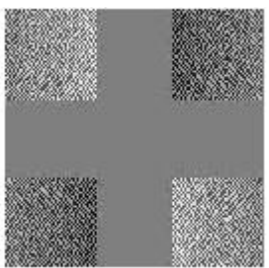

(a)

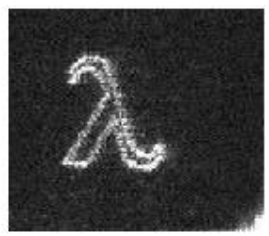

(c)

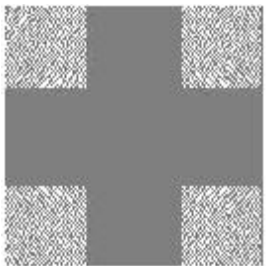

(b)

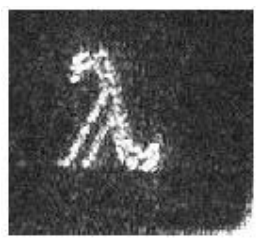

(d)
Figure 10. An examples of compressed spectra calculated numerically by applying our method, compressed spectra in grey level, (b) binary compressed spectra (c) decompressed symbol $(\lambda)$ optically for (a), (d) decompressed binary symbol of $(\lambda)$ for $(b)$.

note that all optical setups had been realized in department of optoelectronic, ISEN-Brest, France.

\section{Conclusions}

In this paper, we have presented a novel method of optical image compression/decompression using a real Fourier plane. This optical compression technique processes the image in whole image at once and does not divide it into blocks, thanks to coherent optics. We successively simplified our earlier optical image compression method OJPEG. The proposed method has shown to be a good compression alternative for all types of images. In the practical part, we proposed two optical architectures: one for compression and another one for decompression. Indeed, we meet several difficulties with set up alignments of laser beam for assuring a multiplication between input plan (displayed over an SLM SEIKO) with low-pass filter whose select the pertinent information displayed over another SLM DisplayTech). In fact, decompressing an image is easy to implement optically where only one SLM is required as demonstrated in Figures $\mathbf{4}$ and 9. According to the above considerations, we successfully proof this novel optical compression/decompression method, reduced the information volume representing an image in pixels. Proposed method has several advantages compared to other algorithms. We can list the main features of proposed method according to obtained results:

- Minimize the time required for compression/decompression by using the principle of coherent optics.

- Gain on space memory.

- Improve the quality of reconstructed image numerically and optically.

- Avoid the problem that characterized digital JPEG as (mosaic effect).

- Simplify our earlier compression method optical JPEG.

- Apply this method in combination between numerical and optical (optical compression and numerical decompression), we can go ahead for all optics. 


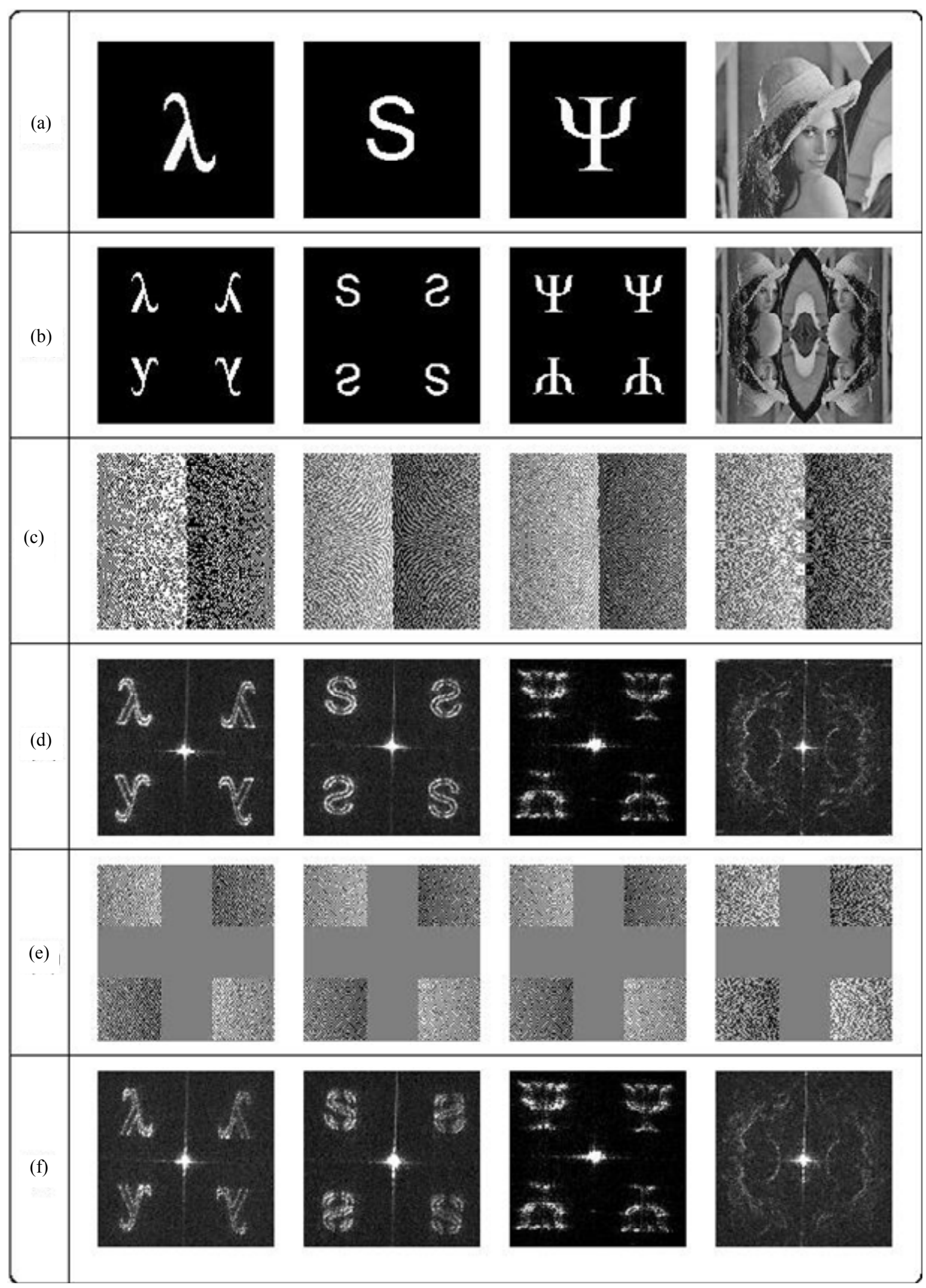

Figure 11. Results of optical compression/decompressionsetup of the real Fourier plane using SEIKO modulator: (a) original image I; (b) replicated image I; (c) replicated spectrum of the image; (d) optical output plane; (e) compressed spectra; (f) decompressed image. 




Figure 12. Validation results of optical compression/decompression implementation using a Display Tech modulator to display the binary compressed spectra, (a) input image I; (b) replicated image I; (c) replicated image spectra; (d) implementation of replicated image spectra; (e) compressed spectra; (f) decompressed image.

Finally the numerical and optical results obtained are generally considered to be more robust as appear in Section 6.

\section{Acknowledgements}

The author wants to thank Prof. Habib Hama (Moncton
University), and Prof. M. Sujr (Sanaa University) for reading this paper and for their nice comments.

\section{REFERENCES}

[1] A. L. Oldenburg, J. J. Reynolds, D. L. Marks and S. A. Boppart, "Fast-Fourier-Domain Delay Line for in Vivo 
Optical Coherence Tomography with a Polygonal Scanner," Applied Optics, Vol. 42, No. 22, 2003, pp. 46064611.

[2] A. Alkholidi, A. Alfalou and H. Hamam, "A New Approach for Optical Colored Image Compression Using the JPEG Standards," Signal Processing, Vol. 87, 2007, pp. 569-583.

[3] A. Alkholidi, "Analyse and Implementation of Compression/Decompression Processors Based on JPEG and JPEG 2000 Standards," Ph.D. Thesis, Université de Bretagne Ocedentale (UBO), Brest, 2007.

[4] A. Alkholidi, A. Cottour, A. Al falou, H. Hamam and G. Keryer, "Real Time Optical 2-D Wavelet Transform Based of the JPEG2000 Standards," European Physical Journal (EPJ), Applied Physics, Vol. 44, 2008, pp. 261272.

[5] A. Alfalou, et al., "Assessing the Performance of a Method of Simultaneous Compression and Encryption of Multiple Images and Its Resistance against Various Attacks," Optics Express, Vol. 21, No. 7, 2013, pp. 80258043. doi:10.1364/OE.21.008025

[6] A. E. Shortt, T. J. Naughton and B. Javidi, "Compression of Optically Encrypted Digital Holograms Using Artificial Neural Networks," Journal of Display Technology,
Vol. 2, No. 4, 2006, pp. 401-410.

[7] C.-H. Chuang and Y.-L. Chen, "Steganographic Optical Image Encryption System Based on Reversible Data Hiding and Double Random Phase Encoding," Optical Engineering, Vol. 52, No. 2, 2013, Article ID: 028201.

[8] X. Q. Zhou, et al., "Spatial-Frequency-Compression Scheme for Diffuse Tomography with Dataset," Applied Optics, Vol. 52, No. 9, 2013, pp. 1779-1792.

[9] H. Wang, S. S. Han and M. Kolobov, "Quantum Limits in Compressed Sensing of Optical Images," Quantum Electronics and Laser Science Conference, San Jose, 6 May 2012.

[10] R. C. Gonzalez and R. E. Woods, "Digital Image Compression," Prentice Hall, 2009.

[11] W. Goodman, "Introduction to Fourier Optics," MacGrawHill, New York, 1968.

[12] Y. Suzuki, "Spatial Light Modulators for Phase-Only Modulation," IEEE, 1999, pp. 1312-1313.

[13] G. Ntogari, et al., "A Numerical Study of Optical Switches and Modulators Based on Ferroelectronic Liquid Crystals," Journal of Optics A: Pure and Applied Optics, Vol. 7, 2004, pp. 82-87. 